\title{
Penfigoide gestacional y erupción ampollar en el recién nacido
}

\section{Bullous pemphygoid and blisters in the newborn}

\author{
María Laura Marchese ${ }^{1}$, María Walker ${ }^{1}$, Alejandro Olivera ${ }^{2}$, Javier Anaya ${ }^{3}$ y Corina Busso ${ }^{4}$
}

\begin{abstract}
RESUMEN
El penfigoide gestacional es una dermatosis rara, que se presenta durante el embarazo. Se caracteriza por una respuesta autoinmune contra las proteínas de los hemidesmosomas, que genera un clivaje entre la epidermis y la dermis tanto de la piel como de las mucosas. Clínicamente, presenta prurito intenso, placas y pápulas eritematosas, que evolucionan

a ampollas con distribución en el abdomen y los miembros. Como complicaciones, en el feto puede generar parto prematuro y bajo peso para la edad gestacional, con alto riesgo de mortalidad.

Palabras clave: dermatosis del embarazo, penfigoide gestacional, enfermedades ampollares.

Dermatol. Argent. 2020, 26 (1): 35-37
\end{abstract}

\section{ABSTRACT}

Gestational pemphygoid is a rare, autoimmune dermatosis that occurs during pregnancy. It is characterized by an autoimmune response against hemidesmosome proteins, generating a cleavage between the epidermis and the dermis in the skin and mucous membranes. Clinically it presents with intense pruritus, plaques and erythematous papules that evolve to blisters that are distributed mainly in the abdomen and limbs. The complications are preterm birth and low weight for gestational age, with high risk of mortality.

Keywords: pregnancy dermatosis, bullous pemphygoid, blister diseases.

Dermatol. Argent. 2020, 26 (1): 35-37

\footnotetext{
'Médica Dermatóloga, Médica de Planta del Servicio de Dermatología

${ }^{2}$ Médico Dermatólogo Pediatra, Médico de Planta del Servicio de Dermatología

${ }^{3}$ Médico Dermatopatólogo, Médico de Planta de Anatomía Patológica

${ }^{4}$ Magíster en Investigaciones Clínicas y Farmacológicas, Médica Dermatóloga, Jefa del Servicio de Dermatología Hospital Universitario Austral, Pilar, Provincia de Buenos Aires, Argentina
}

\section{CASO CLÍNICO}

Una mujer de 41 años, embarazada de 28 semanas (G2C1), consultó por presentar lesiones pruriginosas en el abdomen y la raíz de los miembros, de 7 días de evolución. En el examen físico se observaban placas eritematoedematosas, algunas con bordes vesiculosos, que formaban rosetas y ampollas tensas. Las lesiones se localizaban en el abdomen y la raíz de los miembros (Fotos 1 y 2). No presentaba compromiso de las mucosas. Con el diagnóstico presuntivo de penfigoide gestacional (PG) o de erupción polimorfa del embarazo, se decidió tomar dos biopsias: una para histopatología y otra para inmunofluorescencia direc-
Contacto del autor: María Laura Marchese

Email:mmarches@cas.austral.edu.ar

Fecha de trabajo recibido: 15/11/2019

Fecha de trabajo aceptado: 5/3/2020

Conflicto de interés: los autores declaran que no existe conflicto de interés.

ta (IFD). Se indicó tratamiento con difenhidramina $50 \mathrm{mg} / 8$ horas, clobetasol y meprednisona $20 \mathrm{mg} /$ día. La histopatología informó: ampollas subepidérmicas y marcada eosinofilia perivascular dérmica (Foto 3). La IFD mostró, en el límite entre la epidermis y la dermis, una imagen lineal continua con IgG, C3 y C1q. Con los datos de la clínica, la histopatología y la IFD, se confirmó el diagnóstico de PG y se aumentó la dosis de meprednisona a $60 \mathrm{mg} / \mathrm{día}$, con controles estrictos de glucemia, tensión arterial y examen obstétrico semanal. A la semana 32 de embarazo se encontraba sin lesiones inflamatorias en la piel. Presentó diabetes ges- 


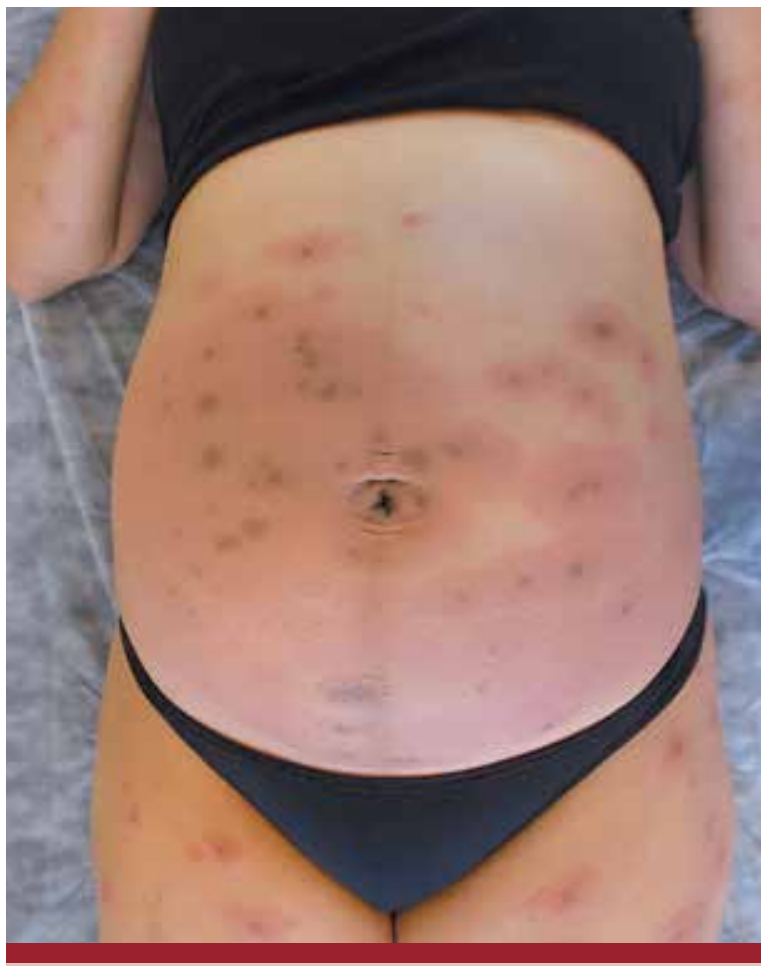

FOTO 1: Placas eritematoedematosas en el abdomen y la raíz de los miembros; algunas vesículas se agrupaban y formaban rosetas.

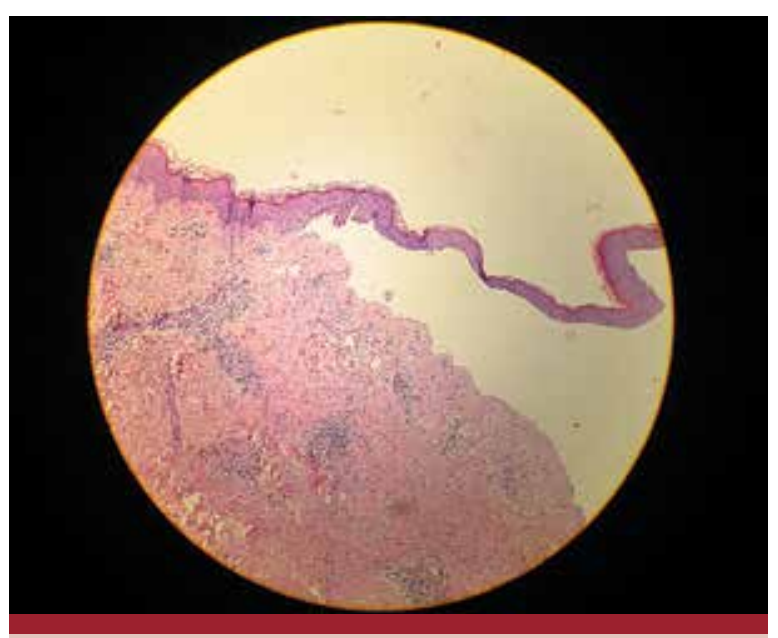

FОT0 3: Se observa la zona de clivaje subepidérmico, infiltrado linfocitario y eosinófilos en la dermis (HyE, 100X).

tacional con requerimiento de insulina. En la semana 38, por considerarlo un embarazo de riesgo debido a la dermatosis y a la diabetes gestacional, se le realizó una cesárea. A las 24 horas de vida, el recién nacido evolucionó con una erupción ampollar generalizada, que se resolvió de manera espontánea a las 48 horas (Foto 4). Dados el antecedente materno y las lesiones ampollares, que se autolimitaron, se interpretó el cuadro como un penfigoide neonatal. A la segunda semana de puerperio se indicó el descenso paulatino de los corticosteroides, con buena evolución. Al cuarto mes posparto la dermatosis se había resuelto.

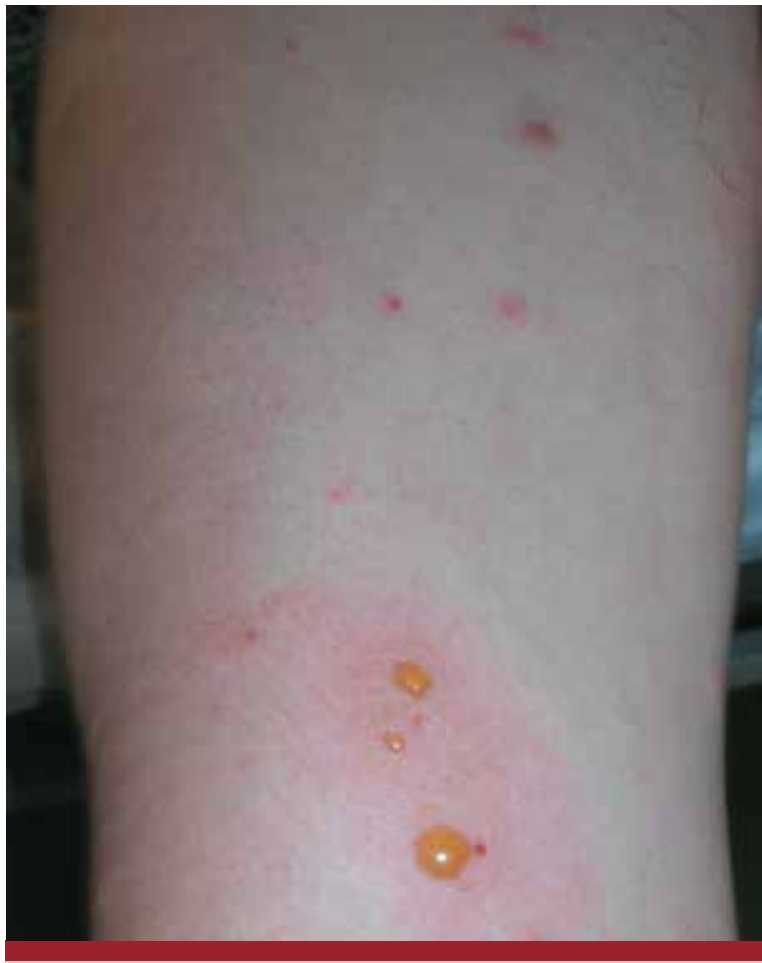

FOTO2: Placas eritematoedematosas con ampollas tensas en los muslos.

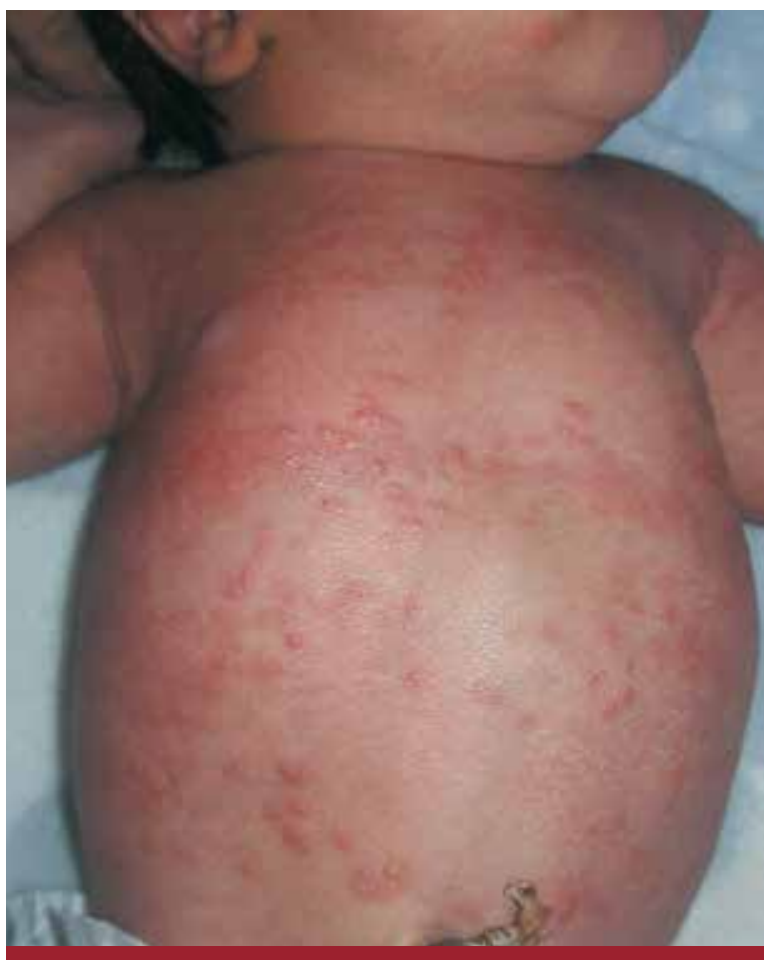

F0T0 4: Erupción ampollar generalizada en el recién nacido.

\section{COMENTARIOS}

Los cambios dermatológicos durante el embarazo pueden dividirse en cambios fisiológicos, alteraciones de dermatosis preexistentes y dermatosis específicas del embarazo $^{1,2}$. 
Las dermatosis específicas del embarazo representan un grupo de dermatosis que ocurren solo durante ese período o el puerperio inmediato. Estas dermatosis son: el PG, la erupción polimorfa del embarazo, la colestasis intrahepática del embarazo y la erupción atópica del embarazo².

La incidencia del PG es de 1/60.000 embarazos. Suele verse en multíparas en el segundo y el tercer trimestre y las recurrencias se producen en un 33-55\% de los $\operatorname{casos}^{3,4}$.

La patogenia es autoinmune; se detectaron anticuerpos contra las proteínas BP 180 (BP Ag1) y BP 230 (BP Ag2) de los hemidesmosomas, con el subsiguiente clivaje entre la epidermis y la dermis. El trofoblasto y las células estromales del amnios muestran una expresión anormal del HLA II, que genera una presentación del BP Ag1 al sistema inmunitario materno. Este antígeno se encuentra en la placenta, en las membranas fetales y en la membrana basal de la piel. A partir de la producción de anticuerpos IgG contra ese antígeno, se origina una respuesta autoinmune con activación del complemento, depósito de inmunocomplejos y quimiotaxis de eosinófilos, que produce un daño tisular con formación de ampollas ${ }^{5,6}$.

En cuanto a las manifestaciones clínicas, el PG presenta prurito intenso y lesiones eritematoedematosas polimorfas. Inicialmente, se observan pápulas y placas urticarianas seguidas de vesículas y ampollas tensas. Las lesiones aparecen en la región umbilical y luego se extienden al resto del abdomen, muslos, palmas y plantas. Las mucosas casi nunca están afectadas ${ }^{7,8}$.

La mayoría de las pacientes presentan remisión durante la etapa final del embarazo y evolucionan con un rebrote en el puerperio inmediato ${ }^{1}$.

El estudio histopatológico y la IFD son necesarios para arribar al diagnóstico. En la etapa inicial, se evidencia edema en la dermis papilar con infiltrados linfocitarios, histiocitarios y eosinofílicos y, en las etapas posteriores, se puede ver la formación de ampollas subepidérmicas. En la IFD se observa depósito de C3 e IgG en la unión dermoepidérmica. El C3 está presente en el 100\% de los casos, mientras que la IgG, solo en un $25-50 \%$. En la inmu-

\section{BIBLIOGRAFÍA}

1. Ambros-Rudolph CM. Dermatoses of pregnancy - clues to diagnosis, fetal risk and therapy. Ann Dermatol 2011;23:265-275.

2. Ambros-Rudolph CM, Müllegger RR, Vaughan-Jones SA, Kerl H, et ál. The specific dermatoses of pregnancy revisited and reclassified: results of a retrospective two-center study on 505 pregnant patients. J Am Acad Dermatol 2006;54:395-404.

3. Al-Saif F, Elisa A, Al-Homidy A, Al-Ageel A, et ál. Retrospective analysis of pemphigoid gestationis in 32 Saudi patients clinicopathological features and a literature review. J Reprod Immunol 2016;116:42-45.

4. Huilaja L, Mäkikallio K, Sormunen R, Lohi J, et ál. Gestational pemphigoid: placental morphology and function. Acta Derm Venereol 2013;93:33-38.

5. Borthwick GM, Sunderland CA, Holmes RC, Black MM, et ál. Abnormal expression of HLA-DR antigen in the placenta of a patient with pemphigoid gestationis. J Reprod Immunol nofluorescencia indirecta (IFI) se detecta IgG anti-MB en el 30-100\% de los casos ${ }^{3}$.

En cuanto a los diagnósticos diferenciales, es importante distinguir esta entidad de las otras dermatosis específicas del embarazo. La erupción atópica del embarazo se presenta principalmente en la primera etapa de la gestación en pacientes con antecedentes de atopia, en tanto que el PG se evidencia sobre todo en el segundo o el tercer trimestre. La erupción polimorfa del embarazo puede ser difícil de diferenciar del PG, ya que ambos presentan inicialmente prurito y placas eritematoedematosas, aunque la primera suele respetar el área umbilical. Para diferenciar estas dos entidades, puede ser necesario realizar una biopsia para histopatología e IFD. En la histopatología de la erupción polimorfa del embarazo se encuentra un infiltrado inflamatorio inespecífico, sin ampollas y la IFD es negativa. La colestasis intrahepática del embarazo presenta prurito, generalmente en el tercer trimestre y se asocia con complicaciones fetales como bajo peso para la edad gestacional, prematuridad, líquido amniótico meconial, hemorragias y muerte fetal. En el laboratorio se detecta un aumento de los ácidos biliares ${ }^{1}$.

El tratamiento se basa en corticosteroides sistémicos. La dosis sugerida es de $0,5 \mathrm{mg} / \mathrm{kg} /$ día de meprednisona, con un descenso paulatino posterior. La mayoría de las pacientes están sin manifestaciones clínicas a los 6 meses del posparto $^{9,10}$.El uso de corticosteroides sistémicos no afecta el pronóstico fetal.

Como complicaciones en el feto, en un 15\% de los casos puede presentarse parto prematuro y bajo peso para la edad gestacional ${ }^{6}$.

En los recién nacidos se ha visto la aparición de lesiones vesiculosas distribuidas en todo el tegumento, entidad conocida como penfigoide neonatal. Esta erupción, descripta en un $10 \%$ de las pacientes, suele autolimitarse ${ }^{6}$.

Consideramos que es importante reconocer esta enfermedad para realizar el diagnóstico precoz, iniciar el tratamiento temprano y diferenciarla de las otras dermatosis del embarazo que no traen aparejados riesgos fetales.

1984;6:393-396.

6. Roth MM. Pregnancy dermatoses diagnosis, management, and controversies. Am J Clin Dermatol 2011;12:25-41.

7. Semkova K, Black M. Pemphigoid gestationis: current insights into pathogenesis and treatment. Eur J Obstet Gynecol Reprod Biol 2009;145:138-144.

8. Hallaji Z, Mortazavi H, Ashtari S, Nikoo A, et ál. Pemphigoid gestationis: clinical and histologic features of twenty three patients. Int J Womens Dermatol 2016;3:86-90.

9. Jenkins RE, Hern S, Black MM. Clinical features and management of 87 patients with pemphigoid gestationis. Clin Exp Dermatol 1999;24:255-259.

10. Jenkins RE, Jones SA, Black MM. Conversion of pemphigoid gestationis to bullous pemphigoid-two refractory cases highlighting this association. Br J Dermatol 1996;135:595-598. 